\title{
Design New Robust Self Tuning Fuzzy Backstopping Methodology
}

\author{
Omid Avatefipour, Farzin Piltan, Mahmoud Reza Safaei Nasrabad, Ghasem Sahamijoo, Alireza Khalilian \\ Institute of Advance Science and Technology, Intelligent control and Robotics Lab. IRAN SSP, Shiraz/Iran, \\ http://WWW.IRANSSP.COM \\ Email: Piltan_f@iranssp.com
}

\begin{abstract}
This research is focused on proposed Proportional-Integral (PI) like fuzzy adaptive backstopping fuzzy algorithms based on ProportionalDerivative (PD) fuzzy rule base with the adaptation laws derived in the Lyapunov sense. Adaptive SISO PI like fuzzy adaptive backstopping fuzzy method has two main objectives; the first objective is design a SISO fuzzy system to compensate for the model uncertainties of the system, and the second objective is focused on the design PI like fuzzy controller based on PD method as an adaptive methodology. Classical backstopping control is robust to control model uncertainties and external disturbances and is a main controller in this research. The fuzzy controller is used in this method to system compensation. To increase the robust of this controller adaptive PI like fuzzy controller is introduced and applied to backstopping fuzzy controller. Classical backstopping control method has difficulty in handling unstructured model uncertainties. One can overcome this problem by combining a backstopping controller and artificial intelligence (e.g. fuzzy logic). To approximate a time-varying nonlinear dynamic system, a fuzzy system requires a large amount of fuzzy rule base. This large number of fuzzy rules will cause a high computation load. The addition of an adaptive law to a backstopping fuzzy controller to online tune the coefficients in use will ensure a moderate computational load. The adaptive laws in this algorithm are designed based on the Lyapunov stability theorem. This method is applied to continuum robot manipulator to have the best performance.
\end{abstract}

Index Terms - Continuum robot manipulator, robust backstopping controller, fuzzy logic system, adaptive methodology, minimum rule base, PI like fuzzy controller.

\section{Introduction and background}

Research about mechanical parts and control methodologies in robotic system is shown; the mechanical design, type of actuators, and type of systems drive play important roles to have the best performance controller. More over types of kinematics chain, i.e., serial Vs. parallel manipulators, and types of connection between link and join actuators, i.e., highly geared systems Vs. direct-drive systems are played important roles to select and design the best acceptable performance controllers[1-11]. A serial link continuum robot is a sequence of joints and links which begins with a base frame and ends with an end-effector. This type of robot manipulators, comparing with the load capacitance is more weightily because each link must be supported the weights of all next links and actuators between the present link and end-effector[12-18]. Serial continuum robot manipulators have been used in medical application, and also in research laboratories. One of the most important classifications in controlling the robot manipulator is how the links have connected to the actuators. This classification divides into two main groups: highly geared (e.g., 200 to 1) and direct drive (e.g., 1 to 1 ). High gear ratios reduce the nonlinear coupling dynamic parameters in robot manipulator. In this case, each joint is modeled the same as SISO systems. In high gear robot manipulators which generally are used in industry, the couplings are modeled as a disturbance for SISO systems. Direct drive increases the coupling of nonlinear dynamic parameters of robot manipulators. This effect should be considered in the design of control systems. As a result some control and robotic researchers' works on nonlinear robust controller design[19-24]. Although most of continuum robot manipulator is high gear and this research focuses on design SISO controller.

In modern usage, the word of control has many meanings, this word is usually taken to mean regulate, direct or command. The word feedback plays a vital role in the advance engineering and science. There are several methods for controlling a robot manipulator, which all of them follow two common goals, namely, hardware/software implementation and acceptable performance. However, the mechanical design of robot manipulator is very important to select the best controller but in general two types schemes can be presented, namely, a joint space control schemes and an operation space control schemes[25-35]. Joint space and operational space control are closed loop controllers which they have been used to provide robustness and rejection of disturbance effect. The main target in joint space controller is to design a feedback controller which the actual motion $\left(\mathrm{q}_{\mathrm{a}}(\mathrm{t})\right)$ and desired motion $\left(\mathrm{q}_{\mathrm{d}}(\mathrm{t})\right.$ ) as closely as possible. This control problem is classified into two main groups. Firstly, transformation the desired motion $\mathrm{X}_{\mathrm{d}}(\mathrm{t})$ to joint variable $\mathrm{q}_{\mathrm{d}}(\mathrm{t})$ by inverse kinematics of robot manipulators[36-47]. This control 
include simple PD control, PID control, inverse dynamic control, Lyapunov-based control, and passivity based control that explained them in the following section. The main target in operational space controller is to design a feedback controller to allow the actual end-effector motion $X_{a}(t)$ to track the desired endeffector motion $X_{d}(t)$. This control methodology requires a greater algorithmic complexity and the inverse kinematics used in the feedback control loop. Direct measurement of operational space variables are very expensive that caused to limitation used of this controller in industrial robot manipulators[40-47]. One of the simplest ways to analysis control of multiple DOF robot manipulators are analyzed each joint separately such as SISO systems and design an independent joint controller for each joint. In this controller, inputs only depends on the velocity and displacement of the corresponding joint and the other parameters between joints such as coupling presented by disturbance input. Joint space controller has many advantages such as one type controllers design for all joints with the same formulation, low cost hardware, and simple structure.

A nonlinear methodology is used for nonlinear uncertain systems (e.g., robot manipulators) to have an acceptable performance. These controllers divided into six groups, namely, feedback linearization (computedtorque control), passivity-based control, sliding mode control (variable structure control), artificial intelligence control, backstopping control and adaptive control[1-20]. In control theory, backstopping is a technique developed circa 1990 by Petar V. Kokotovic and others for designing stabilizing controls for a special class of nonlinear dynamical systems. These systems are built from subsystems that radiate out from an irreducible subsystem that can be stabilized using some other method. Because of this recursive structure, the designer can start the design process at the known-stable system and "back out" new controllers that progressively stabilize each outer subsystem. The process terminates when the final external control is reached. Hence, this process is known as backstopping. Backstopping control is work based on cancelling decoupling and nonlinear terms of dynamics formulation of each link. This method computes the required arm torques using the nonlinear feedback control law.

In recent years, artificial intelligence theory has been used in sliding mode control systems. Neural network, fuzzy logic, and neuron-fuzzy are synergic ally combined with nonlinear classical controller and used in nonlinear, time variant, and uncertainty plant (e.g., robot manipulator). Fuzzy logic controller (FLC) is one of the most important applications of fuzzy logic theory. This controller can be used to control nonlinear, uncertain, and noisy systems. This method is free of some modelbased techniques as in classical controllers. As mentioned that fuzzy logic application is not only limited to the modeling of nonlinear systems [31-47]but also this method can help engineers to design easier controller. Control robot arm manipulators using classical controllers are based on manipulator dynamic model. These controllers often have many problems for modelling. Conventional controllers require accurate information of dynamic model of robot manipulator, but these models are multi-input, multi-output and nonlinear and calculate accurate model can be very difficult. When the system model is unknown or when it is known but complicated, it is difficult or impossible to use classical mathematics to process this model[38-40]. The main reasons to use fuzzy logic technology are able to give approximate recommended solution for unclear and complicated systems to easy understanding and flexible. Fuzzy logic provides a method which is able to model a controller for nonlinear plant with a set of IF-THEN rules, or it can identify the control actions and describe them by using fuzzy rules. It should be mentioned that application of fuzzy logic is not limited to a system that's difficult for modeling, but it can be used in clear systems that have complicated mathematics models because most of the time it can be shortened in design but there is no high quality design just sometimes we can find design with high quality. Besides using fuzzy logic in the main controller of a control loop, it can be used to design adaptive control, tuning parameters, working in a parallel with the classical and non-classical control method [32-39]. The applications of artificial intelligence such as neural networks and fuzzy logic in modelling and control are significantly growing especially in recent years. For instance, the applications of artificial intelligence, neural networks and fuzzy logic, on robot arm control have reported in [40-47].

In various dynamic parameters systems that need to be training on-line adaptive control methodology is used. Adaptive control methodology can be classified into two main groups, namely, traditional adaptive method and fuzzy adaptive method. Fuzzy adaptive method is used in systems which want to training parameters by expert knowledge. Traditional adaptive method is used in systems which some dynamic parameters are known. In this research in order to solve disturbance rejection and uncertainty dynamic parameter, adaptive method is applied to artificial backstopping controller based on PI like fuzzy controller. For nonlinear dynamic systems (e.g., robot manipulators) with various parameters, adaptive control technique can train the dynamic parameter to have an acceptable controller performance. Calculate several scale factors are common challenge in classical backstopping controller and fuzzy logic controller, as a result it is used to adjust and tune coefficient. Research on adaptive nonlinear control is significantly growing, for instance, different adaptive fuzzy controllers have been reported in [40-47]. This paper is organized as follows; second part focuses on the modeling dynamic formulation based on Lagrange methodology, design backstopping control and fuzzy logic methodology. Third part is focused on the methodology which can be used to reduce the error, increase the performance quality and increase the robustness and stability. Simulation result and discussion is illustrated in forth part which based on trajectory following and disturbance rejection. The last 
part focuses on the conclusion and compare between this method and the other ones.

\section{THEORY}

Dynamic Formulation of Continuum Robot: The Continuum section analytical model developed here consists of three modules stacked together in series. In general, the model will be a more precise replication of the behavior of a continuum arm with a greater of modules included in series. However, we will show that three modules effectively represent the dynamic behavior of the hardware, so more complex models are not motivated. Thus, the constant curvature bend exhibited by the section is incorporated inherently within the model. The model resulting from the application of Lagrange's equations of motion obtained for this system can be represented in the form

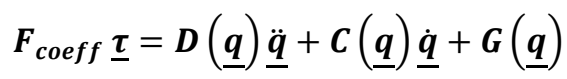

Where ta vector of input forces and $\mathrm{q}$ is is a vector of generalized co-ordinates. The force coefficient matrix $\mathrm{F}_{\text {coeff }}$ transforms the input forces to the generalized forces and torques in the system. The inertia matrix, D is composed of four block matrices. The block matrices that correspond to pure linear accelerations and pure angular accelerations in the system (on the top left and on the bottom right) are symmetric. The matrix $\mathrm{C}$ contains coefficients of the first order derivatives of the generalized co-ordinates. Since the system is nonlinear, many elements of $\mathrm{C}$ contain first order derivatives of the generalized co-ordinates. The remaining terms in the dynamic equations resulting from gravitational potential energies and spring energies are collected in the matrix G. The coefficient matrices of the dynamic equations are given below,

$$
\begin{aligned}
& \text { Fcoeff }= \\
& {\left[\begin{array}{cccccc}
1 & 1 & \cos \left(\theta_{1}\right) & \cos \left(\theta_{1}\right) & \cos \left(\theta_{1}+\theta_{2}\right) & \cos \left(\theta_{1}+\theta_{2}\right) \\
0 & 0 & 1 & 1 & \cos \left(\theta_{2}\right) & \cos \left(\theta_{2}\right) \\
0 & 0 & 0 & 0 & 1 & 1 \\
1 / 2 & -1 / 2 & 1 / 2 & -1 / 2 & 1 / 2+s_{2} \sin \left(\theta_{2}\right) & -1 / 2+s_{2} \sin \left(\theta_{2}\right) \\
0 & 0 & 1 / 2 & -1 / 2 & 1 / 2 & -1 / 2 \\
0 & 0 & 0 & 0 & 1 / 2 & -1 / 2
\end{array}\right]}
\end{aligned}
$$

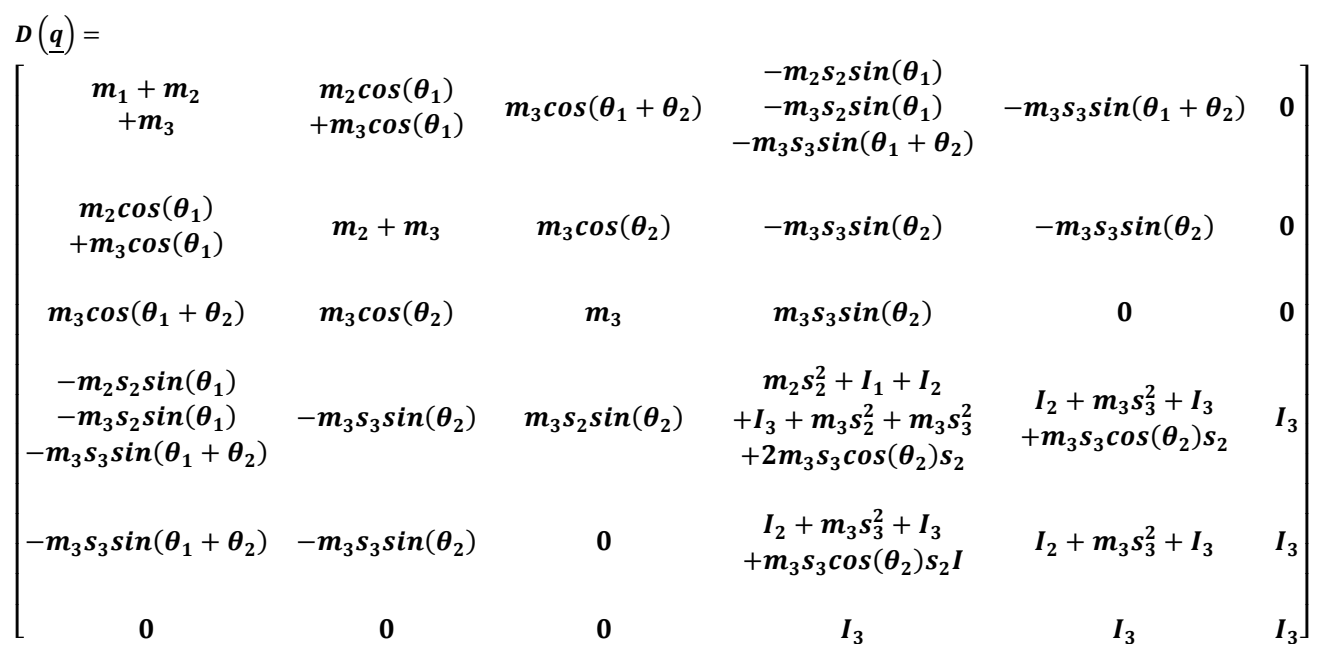




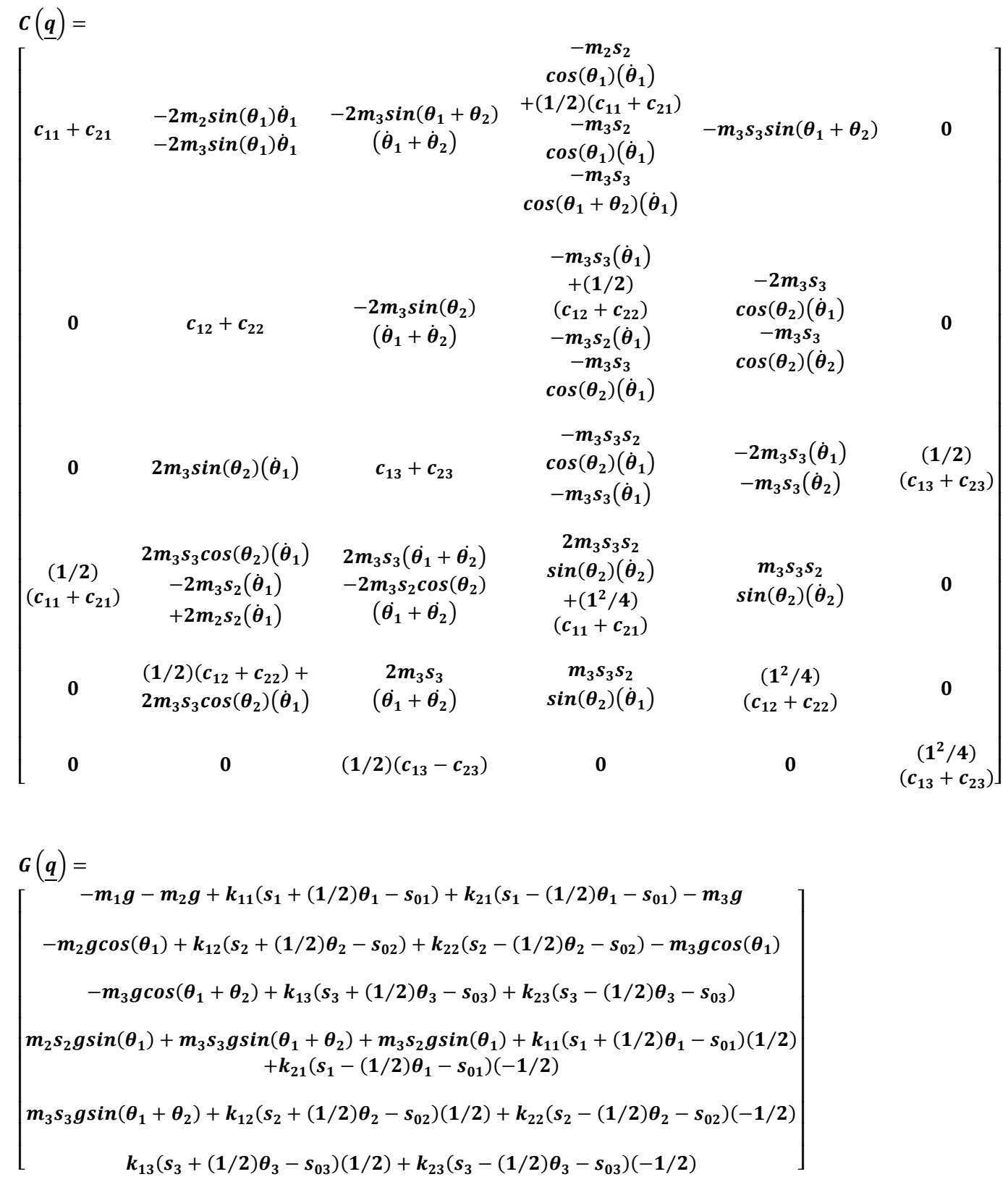

Backstopping Controller: The continuum robot dynamics in (1) have the appropriate structure for the socalled backstopping controller design method. With the position error defined as $\quad Z_{1}=X_{d}-X_{a}$, all joints will track the desired specified state $X_{d}$ if the error dynamics are given as follows:

$\left(\dot{Z}_{1}+\left[K_{p}\right] Z_{1}\right)=0$

where $\left[K_{p}\right]$ is a positive definite gain matrix. The error dynamics in (6) can be rewritten as:

$X_{2}=\dot{X}_{d}+\left[K_{p}\right] Z_{1}$

Substitution of (7) into (1) makes the position error dynamics go to zero. Since the state vector $x_{2}$ is not a control variable, (7) cannot be directly substituted into (1). The expression in (7) is therefore defined as a fictitious control input and is labeled expressed below as $\mathrm{X}_{2 \mathrm{~d}}$.

$\boldsymbol{X}_{\mathbf{2}_{d}}=\dot{\boldsymbol{X}}_{\mathbf{1}_{\boldsymbol{d}}}+\left[\boldsymbol{K}_{\boldsymbol{p}}\right]\left(\mathbf{X}_{\mathrm{d}}-\mathbf{X}_{\mathrm{a}}\right)$

The fictitious control input in (8) is selected as the specified velocity trajectory and hence the velocity error can be defined as $\mathrm{Z}_{2}=\mathrm{X}_{2_{\mathrm{d}}}-\mathrm{X}_{2_{\mathrm{a}}}$. With the following dynamics

$\left(\dot{Z}_{2}+\left[K_{p}\right] Z_{2}\right)=0$

the joint position error will approach zero asymptotically, which will lead to the eventual asymptotic convergence 
of the joint position error. The error dynamics in (9) can be rewritten as:

$X_{2}=\dot{X}_{d}+\left[K_{p}\right] Z_{2}$

Substitution of (9) into (1) leads to the following expression as the desired stabilizing torque:

$\tau=[H]\left(\dot{X}_{2_{d}}+\left[K_{p}\right] Z_{2}\right)+C\left(X_{1}, X_{2}\right)$

The desired torque control input is a nonlinear compensator since it depends on the dynamics of the spherical motor. The time derivative of desired velocity vector is calculated using (9). In terms of the desired state trajectory, and its time derivatives and the position and velocity state variables, the desired torque can be rewritten in following form:

$\tau=[H] y+C\left(X_{1}, X_{2}\right)$

Where

$$
\begin{array}{r}
y=\ddot{X}_{1_{d}}+\left(\left[K_{p}\right]+\left[K_{d}\right]\right)\left(\dot{X}_{1_{d}}-\dot{X}_{1}\right) \\
+\left(\left[K_{p}\right]\left[K_{d}\right] \mathbf{X}_{\mathbf{d}}-\mathbf{X}_{\mathbf{a}}\right.
\end{array}
$$

The backstopping controller developed above is very similar to inverse dynamics control algorithm developed for robotic manipulators. The backstopping controller is ideal from a control point of view as the nonlinear dynamics of the continuum robot are cancelled and replaced by linear subsystems. The drawback of the backstopping controller is that it requires perfect cancellation of the nonlinear continuum robot dynamics. Accurate real time representations of the robot dynamics are difficulty due to uncertainties in the system dynamics resulting from imperfect knowledge of the robot mechanical parameters; existence of unmolded dynamics and dynamic uncertainties due to payloads. The requirement for perfect dynamic cancellation raises sensitivity and robustness issues that are addressed in the design of a robust backstopping controller. Another drawback of the backstopping controller is felt during real-time implementation of the control algorithm. Implementation of the backstopping controller requires the computation of the exact robot dynamics at each sampling time. This computational burden has an effect on the performance of the control algorithm and imposes constraints on the hardware/software architecture of the control system. By only computing the dominant parts of the robot dynamics, this computational burden can be reduced. These drawbacks of the backstopping controller makes it necessary to consider control algorithms that compensate for both model uncertainties and for approximations made during the on-line computation of robot dynamics. The next section provides robust modifications of the backstopping controller described in this section. Fig. 1 shows the block diagram of backstopping controller.

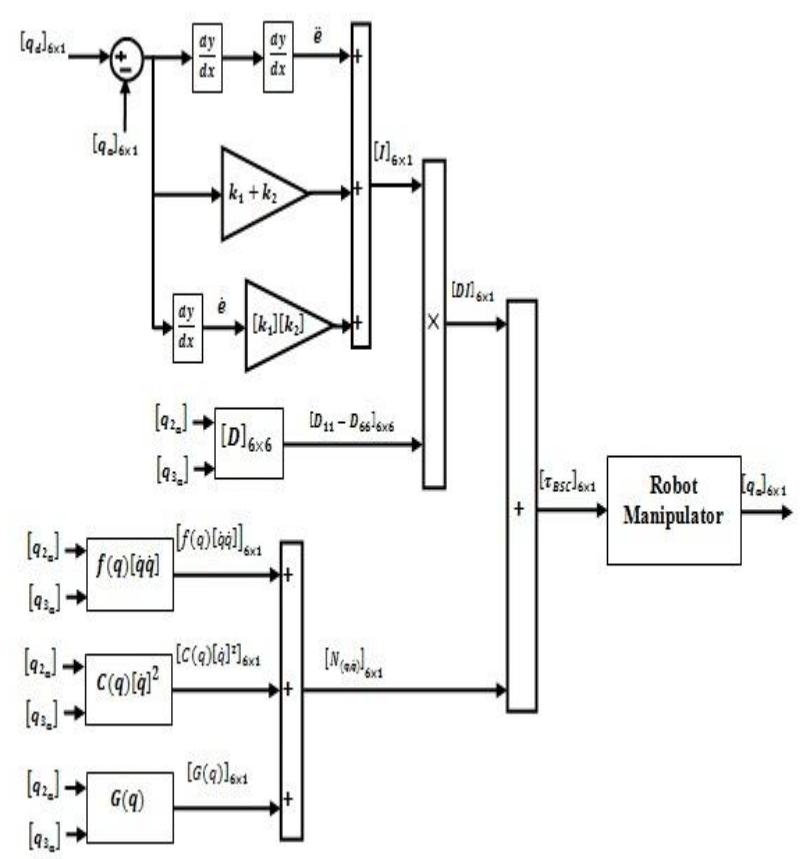

Figure 1: Block diagram of robust backstopping controller

Robust Backstopping Control: When there are uncertainties in the spherical motor dynamics due to modeling inaccuracy and computational relaxation, robust controllers are ideal for ensuring system stability. When the system dynamics are completely known, the required torque control vector for the control of the spherical motor is given by (12) and (13). In the presence of modeling uncertainties, a reasonable approximation of the torque control input vector is given by

$T_{d}=[\widehat{H}] \boldsymbol{y}+\widehat{C}$

Where $[\widehat{H}]$ and $\hat{C}$ are estimates of the inertia and coriolis terms in the spherical motor dynamics; and $y$ is given by (13). The uncertainty on the estimates are expressed as

$[\widetilde{\boldsymbol{H}}]=[\widehat{\boldsymbol{H}}]-[\boldsymbol{H}]$

$[\widetilde{\boldsymbol{C}}]=[\widehat{\boldsymbol{C}}]-[\boldsymbol{C}]$

These uncertainties account for both imperfect modeling and intentional computational simplification. Application of the approximate control vector given by (14) leads to the following expression for the closed loop dynamics:

$[\boldsymbol{H}] \dot{x}_{2}+\boldsymbol{C}=[\widehat{H}] \boldsymbol{y}+\widehat{C}$

Since the inertia matrix $[\mathrm{H}]$ is symmetric and positive definite, the closed loop dynamics in (17) can be rewritten as 
$\ddot{x}_{1}=y-\eta$

Where

$\boldsymbol{\eta}=\left([\boldsymbol{I}]-[\boldsymbol{H}]^{-1}[\widehat{H}]\right) y-[H]^{-1} \widetilde{\boldsymbol{C}}$

Substitution of (13) for $\boldsymbol{y}$ in (18) results in the following expression for the closed loop error dynamics.

$\ddot{\widetilde{x}}_{1}+\left(\left[K_{p}\right]+\left[K_{d}\right]\right) \dot{\tilde{x}}_{1}+\left[K_{p}\right]\left[K_{d}\right] \widetilde{x}_{1}=\eta$

Defining a new error state vector,

$\xi=\left[\begin{array}{c}\widetilde{x}_{1} \\ \dot{\widetilde{x}}_{1}\end{array}\right]$

the error dynamics in (19) can be expressed as

$\dot{\xi}=[F] \xi+[D]\left(\ddot{\mathbf{x}}_{1 \mathrm{~d}}-\mathbf{y}+\boldsymbol{\eta}\right)$

Where $[\boldsymbol{F}]=\left[\begin{array}{ll}{[\mathbf{0}]} & {[\mathbf{1}]} \\ {[\mathbf{0}]} & {[\mathbf{0}]}\end{array}\right]$ and $[\boldsymbol{D}]=\left[\begin{array}{l}{[\mathbf{0}]} \\ {[\mathbf{1}]}\end{array}\right]$ are block matrices of dimensions $R^{6 \times 6}$ and $R^{6 \times 3}$ respectively. Since $\boldsymbol{\eta}$ is a nonlinear function of the position and velocity state vectors, the system error dynamics in the above equation are nonlinear and coupled. The backstopping controller developed in the previous section cannot guarantee system stability. The Lyapunov direct method is, however, used to design an outer feedback loop on the error dynamics that compensates for the system uncertainty contributed by $\boldsymbol{\eta}$.

Fuzzy Inference Engine: This section provides a review about foundation of fuzzy logic based on [15-20]. Supposed that $U$ is the universe of discourse and $x$ is the element of $U$, therefore, a crisp set can be defined as a set which consists of different elements $(x)$ will all or no membership in a set. A fuzzy set is a set that each element has a membership grade, therefore it can be written by the following definition;

$\boldsymbol{A}=\left\{\boldsymbol{x}, \boldsymbol{\mu}_{\boldsymbol{A}}(\boldsymbol{x}) \mid \boldsymbol{x} \in \boldsymbol{X}\right\} ; \boldsymbol{A} \in \boldsymbol{U}$

Where an element of universe of discourse is $x, \mu_{A}$ is the membership function (MF) of fuzzy set. The membership function $\left(\mu_{A}(x)\right)$ of fuzzy set $A$ must have a value between zero and one. If the membership function $\mu_{A}(x)$ value equal to zero or one, this set change to a crisp set but if it has a value between zero and one, it is a fuzzy set. Defining membership function for fuzzy sets has divided into two main groups; namely; numerical and functional method, which in numerical method each number has different degrees of membership function and functional method used standard functions in fuzzy sets. The membership function which is often used in practical applications includes triangular form, trapezoidal form, bell-shaped form, and Gaussian form.
Linguistic variable can open a wide area to use of fuzzy logic theory in many applications (e.g., control and system identification). In a natural artificial language all numbers replaced by words or sentences.

If - then Rule statements are used to formulate the condition statements in fuzzy logic. A single fuzzy If - then rule can be written by

\section{If $x$ is $A$ Then $y$ is $B$}

Where $A$ and $B$ are the Linguistic values that can be defined by fuzzy set, the If - part of the part of " $x$ is $A$ " is called the antecedent part and the thenpart of the part of " $y$ is $B$ " is called the Consequent or Conclusion part. The antecedent of a fuzzy if-then rule can have multiple parts, which the following rules shows the multiple antecedent rules:

\section{if $e$ is $N B$ and $\dot{e}$ is ML then $T$ is LL}

Where $e$ is error, $\dot{e}$ is change of error, $N B$ is Negative Big, $M L$ is Medium Left, $T$ is torque and $L L$ is Large Left. If - then rules have three parts, namely, fuzzify inputs, apply fuzzy operator and apply implication method which in fuzzify inputs the fuzzy statements in the antecedent replaced by the degree of membership, apply fuzzy operator used when the antecedent has multiple parts and replaced by single number between 0 to 1, this part is a degree of support for the fuzzy rule, and apply implication method used in consequent of fuzzy rule to replace by the degree of membership. The fuzzy inference engine offers a mechanism for transferring the rule base in fuzzy set which it is divided into two most important methods, namely, Mamdani method and Sugeno method. Mamdani method is one of the common fuzzy inference systems and he designed one of the first fuzzy controllers to control of system engine. Mamdani's fuzzy inference system is divided into four major steps: fuzzification, rule evaluation, aggregation of the rule outputs and defuzzification. Michio Sugeno uses a singleton as a membership function of the rule consequent part. The following definition shows the Mamdani and Sugeno fuzzy rule base

Mamdani $F . R^{1}:$ if $x$ is $A$ and
$y$ is $B$ then is $C$
Sugeno F. $R^{1}:$ if $x$ is $A$ and
$y$ is $B$ then $f(x, y)$ is $C$

When $x$ and $y$ have crisp values fuzzification calculates the membership degrees for antecedent part. Rule evaluation focuses on fuzzy operation $(A N D / O R)$ in the antecedent of the fuzzy rules. The aggregation is used to calculate the output fuzzy set and several methodologies can be used in fuzzy logic controller aggregation, namely, Max-Min aggregation, Sum-Min aggregation, Max-bounded product, Max-drastic product, Max-bounded sum, Max-algebraic sum and Min-max. 
Two most common methods that used in fuzzy logic controllers are Max-min aggregation and Sum-min aggregation. Max-min aggregation defined as below

$$
\begin{aligned}
& \mu_{U}\left(x_{k}, y_{k}, U\right)=\mu_{\cup_{i=1}^{r} F R^{i}}\left(x_{k}, y_{k}, U\right) \\
& =\max \left\{\min _{i=1}^{r}\left[\mu_{R_{p q}}\left(x_{k}, y_{k}\right), \mu_{p_{m}}(U)\right]\right\}
\end{aligned}
$$

The Sum-min aggregation defined as below

$$
\begin{aligned}
& \mu_{U}\left(x_{k}, y_{k}, U\right)=\mu_{\cup_{i=1}^{r} F R^{i}}\left(x_{k}, y_{k}, U\right) \\
& =\sum \min _{i=1}^{r}\left[\mu_{R}\left(x_{k}, y_{k}\right), \mu_{p_{m}}(U)\right]
\end{aligned}
$$

Where $r$ is the number of fuzzy rules activated by $x_{k}$ and $y_{k}$ and also $\mu_{\cup_{i=1}^{r} F R^{i}}\left(x_{k}, y_{k}, U\right)$ is a fuzzy interpretation of $i-t h$ rule. Defuzzification is the last step in the fuzzy inference system which it is used to transform fuzzy set to crisp set. Consequently defuzzification's input is the aggregate output and the defuzzification's output is a crisp number. Centre of gravity method $(C O G)$ and Centre of area method $(C O A)$ are two most common defuzzification methods, which COG method used the following equation to calculate the defuzzification

$\operatorname{COG}\left(x_{k}, y_{k}\right)=\frac{\sum_{i} U_{i} \sum_{j=1}^{r} \cdot \mu_{u}\left(x_{k}, y_{k}, U_{i}\right)}{\sum_{i} \sum_{j=1}^{r} \cdot \mu_{u}\left(x_{k}, y_{k}, U_{i}\right)}$

and COA method used the following equation to calculate the defuzzification

$\operatorname{COA}\left(x_{k}, y_{k}\right)=\frac{\sum_{i} U_{i} \cdot \mu_{u}\left(x_{k}, y_{k}, U_{i}\right)}{\sum_{i} \mu_{U}\left(x_{k}, y_{k}, U_{i}\right)}$

Where $\operatorname{COG}\left(x_{k}, y_{k}\right)$ and $\operatorname{COA}\left(x_{k}, y_{k}\right)$ illustrates the crisp value of defuzzification output, $U_{i} \in U$ is discrete element of an output of the fuzzy set, $\mu_{U} \cdot\left(x_{k}, y_{k}, U_{i}\right)$ is the fuzzy set membership function, and $r$ is the number of fuzzy rules.

Based on foundation of fuzzy logic methodology; fuzzy logic controller has played important rule to design nonlinear controller for nonlinear and uncertain systems [20-32]. However the application area for fuzzy control is really wide, the basic form for all command types of controllers consists of:

- Input fuzzification (binary-to-fuzzy[B/F]conversion)

- Fuzzy rule base (knowledge base)

- Inference engine

- Output defuzzification (fuzzy-to-binary

$[\mathrm{F} / \mathrm{B}]$ conversion).

Fig 2 shows the block diagram of Fuzzy inference engine.

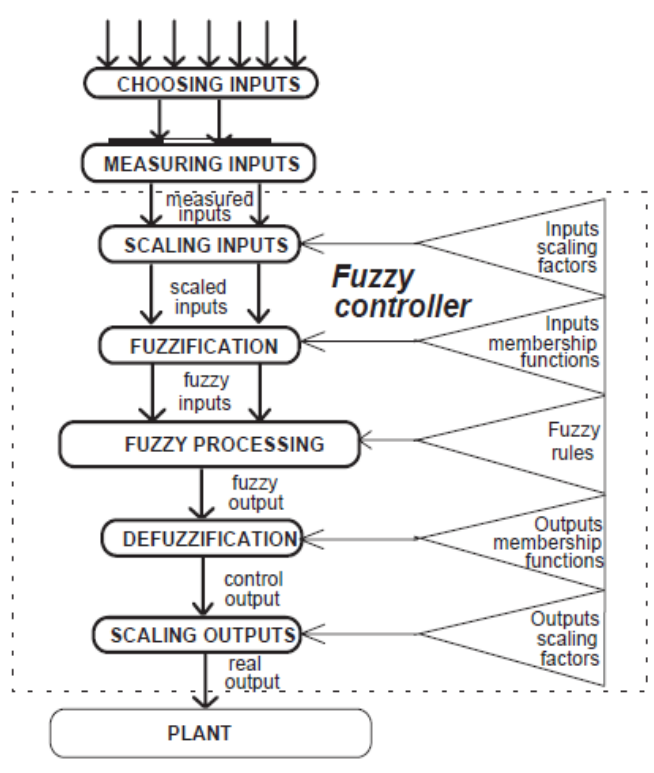

Figure 2: Fuzzy Controller operation

\section{Methodology}

This step is focused on the design minimum rule base Mamdani's backstopping fuzzy controller with application to continuum robot manipulator. As mentioned above pure backstopping controller has nonlinear dynamic parameters limitation in presence of uncertainty and external disturbances. In order to solve this challenge fuzzy inference system is serially applied to backstopping controller. The backstopping method is based on mathematical formulation which this method is introduced new variables into it in form depending on the dynamic equation of continuum robot arm. This method is used as feedback linearization in order to solve nonlinearities in the system. Backstopping controller is divided into two main parts; linear part and nonlinear dynamic formulation. The backstopping controller for continuum robot is calculated by;

$U_{B . S}=U_{e q}+$ D.I

Where $\boldsymbol{U}_{\boldsymbol{B} . \boldsymbol{S}}$ is backstopping output function, $\boldsymbol{U}_{\boldsymbol{e q}} \boldsymbol{q}_{\boldsymbol{B} . \boldsymbol{S}}$ is backstopping nonlinear equivalent function which can be written as (49) and $\boldsymbol{I}$ is backstopping control law which calculated by (1)

$\boldsymbol{U}_{e q_{B . S}}=[\boldsymbol{f}+\boldsymbol{C}+\boldsymbol{G}]$

We have

$I=\left[\ddot{e}+\left(K_{1}+K_{2}\right) \times e+\left(K_{1} \times K_{2}\right) \cdot \dot{e}\right]$

Based on above formulation, FIS in this research has an input $\left(\boldsymbol{N}(\boldsymbol{q}, \dot{\boldsymbol{q}})\right.$ and an output $\left(\boldsymbol{U}_{\boldsymbol{f u z z} \boldsymbol{y}}\right)$, Fig. 3 shows fuzzy inference system. 


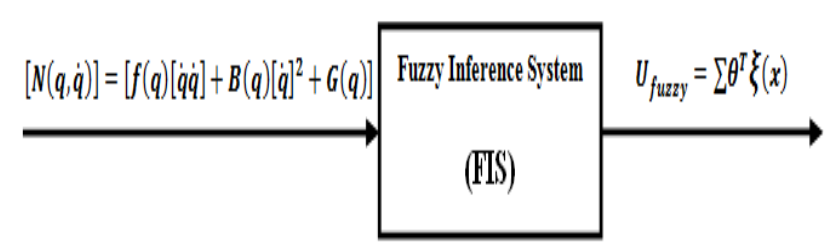

Figure 3 Fuzzy Inference System's input and output

$U_{f u z z y}=\sum_{l=1}^{M} \theta^{T} \zeta(x)$

To estimate the nonlinear term of backstopping controller fuzzy inference system is used in serial with nonlinear dynamic part

$U_{e q B . S_{f u z z y}}=[(f+C+G)] \times \sum_{l=1}^{M} \theta^{T} \zeta(x)$

Based on above formulation the formulation of backstopping fuzzy control is;

$U_{B . S}=U_{e q B . S_{f u z z y}}+D . I$

Most robust control designs are based on the assumption that even though the uncertainty vector $\boldsymbol{\eta}$ is unknown, some information is available on its bound. In designing the robust backstopping controller, a robust term that compensates for the uncertainty in the system dynamics is added to the control law in (50) and (51). Figure 4 shows new backstopping fuzzy controller to modify the nonlinear term of dynamic formulation in uncertain condition.

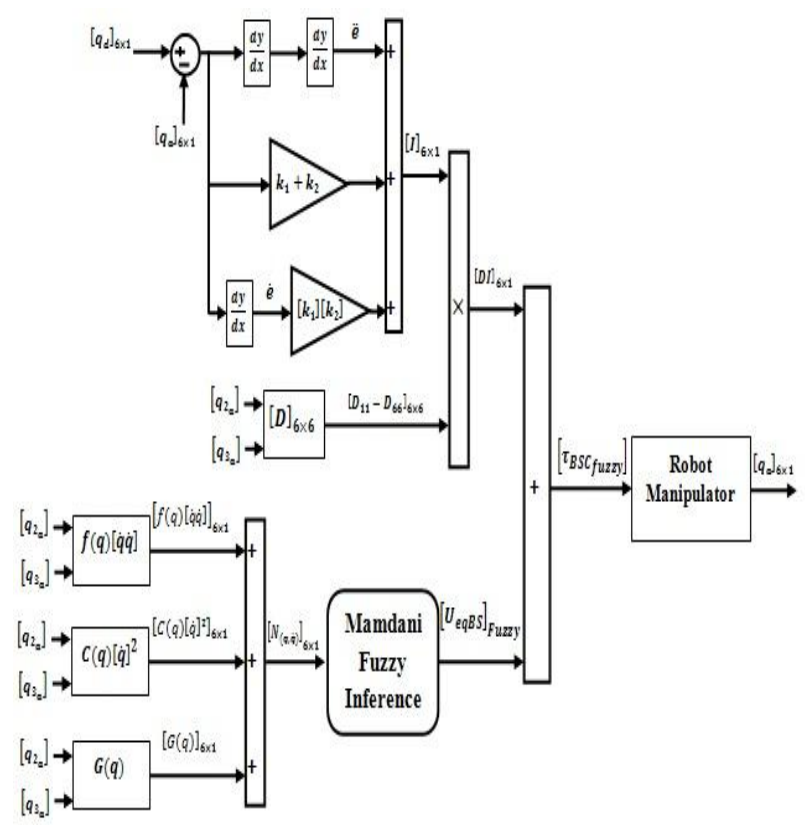

Figure 4: Block diagram of backstopping fuzzy controller for continuum robot
Backstopping fuzzy controller is robust and stable but in presence of unlimited uncertainty. To solve this challenge online tuning controller is design based on PI fuzzy logic controller to tune the coefficient of backstopping controller, $\left(K_{1}\right.$ and $\left.K_{2}\right)$. In this theory the behavior and dynamic of controller is defined by rule base. However defined and number of rule base play important role to design high quality controller but system has limitation to the number of rule base to implementation and the speed of response. Based on literature PI controller can reduce or eliminate the steady state error and design stable controller is the main objective in control theory. To design PI like fuzzy controller if any input is described with seven linguistic values, and any rule has two conditions we will need $7 \times$ $7=49$ rules. The PI controller has two inputs and two coefficients. In PD like fuzzy controller error and change of error are the inputs and if any input is described with seven linguistic values, and any rule has two conditions we will need $7 \times 7=49$ rules. Table 1 shows the rule table of PD like fuzzy controller based on seven linguistic variables for inputs and totally 49 rules.

TABLE 1: Rule Table of PD like Fuzzy Controller

\begin{tabular}{|c|c|c|c|c|c|c|c|}
\hline$e^{\Delta e}$ & PB & PM & PS & Z & NS & NM & NB \\
\hline PB & NB & NB & NB & NB & NM & NS & $\mathbf{Z}$ \\
\hline PM & NB & NB & NB & NM & NS & $\mathbf{Z}$ & PS \\
\hline PS & NB & NB & NM & NS & $\mathbf{Z}$ & PS & $\mathbf{P M}$ \\
\hline $\mathbf{Z}$ & NB & NM & NS & $\mathbf{Z}$ & PS & PM & PB \\
\hline NS & NM & NS & $\mathbf{Z}$ & PS & PM & PB & PB \\
\hline NM & NS & $\mathbf{Z}$ & PS & PM & PB & PB & PB \\
\hline NB & $\mathbf{Z}$ & PS & PM & PB & PB & PB & PB \\
\hline
\end{tabular}

This table includes 49 rules. We are taking into account now not just the error but the change-of-error as well. It allows describing the dynamics of the controller. To explain how this rules set works and how to choose the rules, let us divide the set of all rules into the following five groups:

Group 1: In this group of rules both $\boldsymbol{e}$ and $\boldsymbol{\Delta} \boldsymbol{e}$ are (positive or negative) small or zero. This means that the current value of the process output variable has deviated from the desired level (the set-point) but is still close to it. Because of this closeness the control signal should be zero or small in magnitude and is intended to correct small deviations from the set-point. Therefore, the rules in this group are related to the steady-state behavior of the process. The change-of-error, when it is Negative Small or Positive Small, shifts the output to negative or positive region, because in this case, for example, when $\boldsymbol{e}(\boldsymbol{t})$ and $\Delta \boldsymbol{e}(\boldsymbol{t})$ are both Negative Small the error is already negative and, due to the negative change-oferror, tends to become more negative. To prevent this trend, one needs to increase the magnitude of the control output. 
Group 2: For this group of rules $\boldsymbol{e}(\boldsymbol{t})$ is Positive Big or Medium which implies that actual input is significantly above the set point. At the same time since $\boldsymbol{\Delta} \boldsymbol{e}(\boldsymbol{t})$ is negative, this means that actual input is moving towards the set-point. The control signal is intended to either speed up or slow down the approach to the set-point. For example, if actual input is much below the set-point $(\boldsymbol{e}(\boldsymbol{t})$ is Positive Big) and it is moving towards the setpoint with a small step $(\boldsymbol{\Delta e}(\boldsymbol{t})$ is Negative Small) then the magnitude of this step has to be significantly increased ( $\boldsymbol{U}$ is Negative Medium). However, when actual input is still much below the set-point $(\boldsymbol{e}(\boldsymbol{t})$ is Positive Big) but it is moving towards the set-point very fast $(\boldsymbol{\Delta} \boldsymbol{e}(\boldsymbol{t})$ is Negative Big) no control action can be recommended because the error will be compensated due to the current trend.

Group 3: For this group of rules actual output is either close to the set-point $(\boldsymbol{e}(\boldsymbol{t})$ is Positive Small, Zero, Negative Small) or significantly above it (Negative Medium, Negative Big). At the same time, since $\Delta \boldsymbol{e}(\boldsymbol{t})$ is negative, actual input is moving away from the setpoint. The control here is intended to reverse this trend and make actual input, instead of moving away from the set-point, start moving towards it. So here the main reason for the control action choice is not just the current error but the trend in its change.

Group 4: For this group of rules $\boldsymbol{e}(\boldsymbol{t})$ is Negative Medium or Big, which means that actual input is significantly below the set-point. At the same time, since $\boldsymbol{\Delta} \boldsymbol{e}(\boldsymbol{t})$ is positive, actual input is moving towards the set-point. The control is intended to either speed up or slow down the approach to the set-point. For example, if actual input is much above the set-point $(\boldsymbol{e}(\boldsymbol{t})$ is Negative Big) and it is moving towards the set-point with a somewhat large step ( $\boldsymbol{\Delta} \boldsymbol{e}(\boldsymbol{t})$ is Positive Medium), then the magnitude of this step has to be only slightly enlarged (output is Negative Small).

Group 5: The situation here is similar to the Group 3 in some sense. For this group of rules $\boldsymbol{e}(\boldsymbol{t})$ is either close to the set-point (Positive Small, Zero, Negative Small) or significantly above it (Positive Medium, Positive Big). At the same time since $\boldsymbol{\Delta e}(\boldsymbol{t})$ is positive actual input is moving away from the set-point. This control signal is intended to reverse this trend and make actual input instead of moving away from the set-point start moving towards it. The PD like fuzzy controller shows in Figure 5.

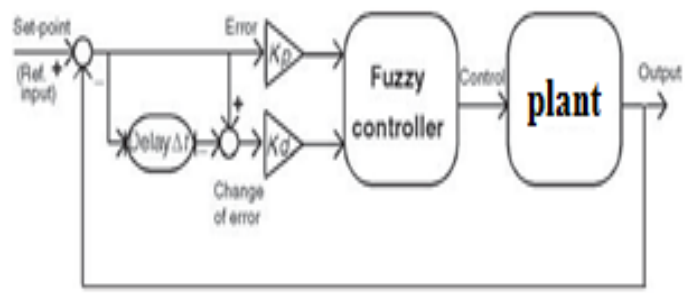

Figure 5: Block diagram of PD like Fuzzy Controller
In PI like fuzzy controller error and integral of error are the inputs and if any input is described with seven linguistic values, and any rule has two conditions we will need $7 \times 7=49$ rules. Figure 6 shows the block diagram of PI like fuzzy controller.

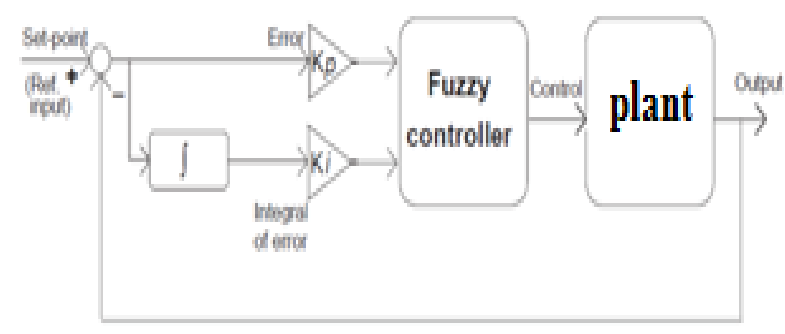

Figure 6: Block diagram of PI like Fuzzy Controller

Now the fuzzy controller and the rules table have other inputs. It means that the rules themselves should be reformulated. Sometimes it is difficult to formulate rules depending on an integral error, because it may have the very wide universe of discourse. We can move the integration from the part proceeding to a fuzzy controller to the part following it. We can integrate the output of a controller, not the input. Then we may have the error and the change of error inputs and still realize the PI-control. Figure 7 shows the other form of PI like fuzzy controller.

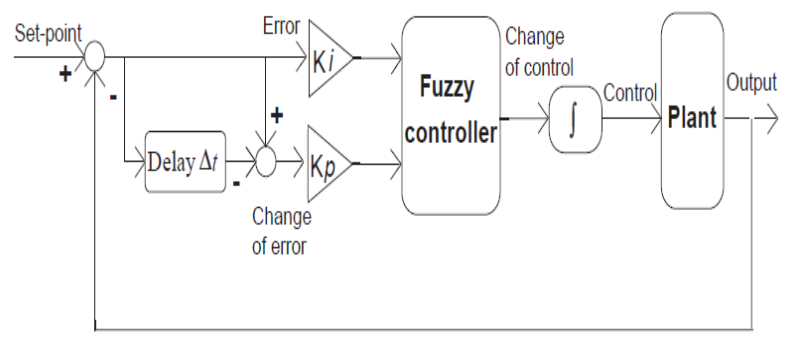

Figure 7: Block diagram of New Type PI like Fuzzy Controller

In this type of PI like fuzzy controller the rule table in PD like fuzzy controller is also used in this system. The PI like fuzzy controller's output is used to tune the backstopping fuzzy controller. To reduce the error and increase the stability and robust adaptive methodology is used as a follows;

$$
\begin{aligned}
F(q, \dot{q}) & =\ominus^{1^{T}} \varepsilon(q, \dot{q}) \\
& =\left[\theta_{1}^{1^{T}} \varepsilon(q, \dot{q}), \ldots, \theta_{m}^{1^{T}} \varepsilon(q, \dot{q})\right]^{T}
\end{aligned}
$$

The adaptation law is given by

$$
\dot{\theta}_{j}^{1}=-\Gamma_{1 j} s_{j} \varepsilon(q, \dot{q})
$$

Where $j=1, \ldots, m$ and $\Gamma_{1 j}-\Gamma_{3 j}$ are positive diagonal matrices.

Figure 8 shows the proposed methodology block diagram applied to continuum robot. 


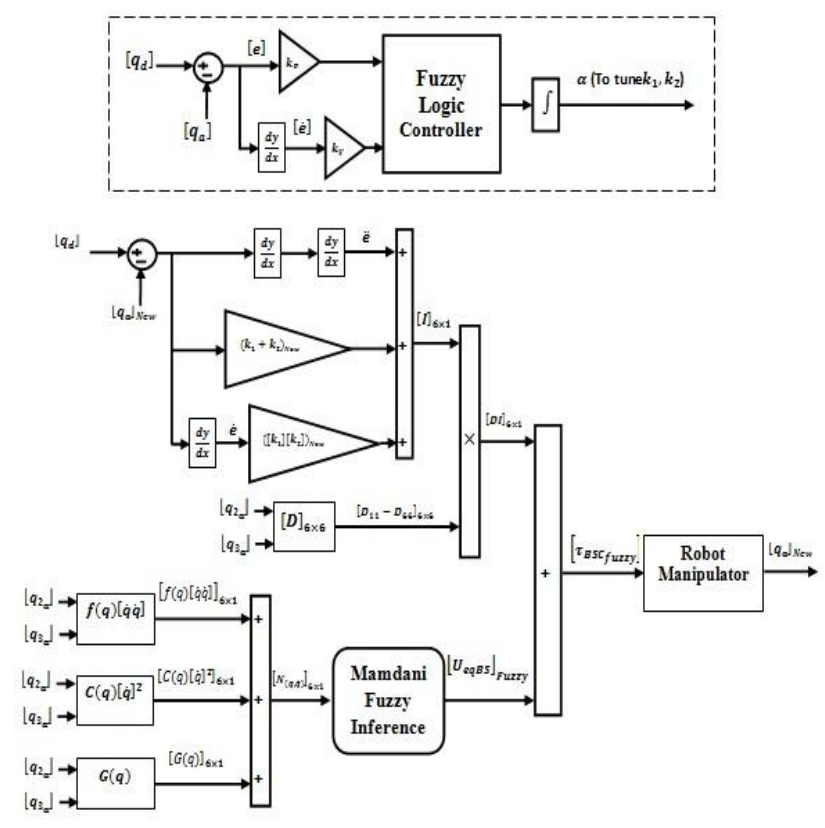

Figure 8: Block diagram of proposed methodology

\section{Result and Discussion}

Backstopping controller (BSC) and adaptive SISO modify PI fuzzy like backstopping fuzzy controller (proposed) are implemented in MATLAB/SIMULINK environment. Tracking performance and disturbance rejections are compared.

Tracking performances: In proposed controller; the performance is depended on three important parameters; nonlinear equivalent part, fuzzy controller and PI like online tuning fuzzy logic controller. According to the following graph proposed controller has accept performance in certain parameters but pure backstopping controller has overshoot about $1 \%$ and steady state error is about 0.05. Based on Fig 9, pure backstopping controller can eliminate the oscillation, it is stable, but it has steady state error. To solve this challenge online tuning based on PI like fuzzy controller is introduced and also the output gain have got to be reduce. In this design rise time in conventional controller is lower than proposed method.

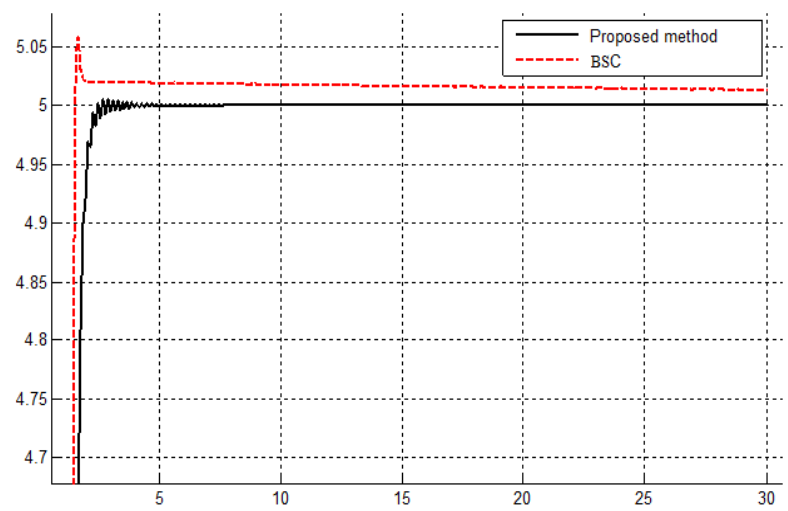

Figure 9: BSC Vs. Proposed Method: applied to robot manipulator
Disturbance rejection: Figure 10 shows the power disturbance elimination in proposed method and conventional backstopping controller in presence of external disturbance and uncertainty parameters. The disturbance rejection is used to test and analyzed the robustness comparisons of these controllers for step trajectory. A band limited white noise with predefined of $40 \%$ the power of input signal value is applied to the step trajectory. According to the following graph, pure backstopping controller has moderate fluctuation in presence of external disturbance and uncertainty.

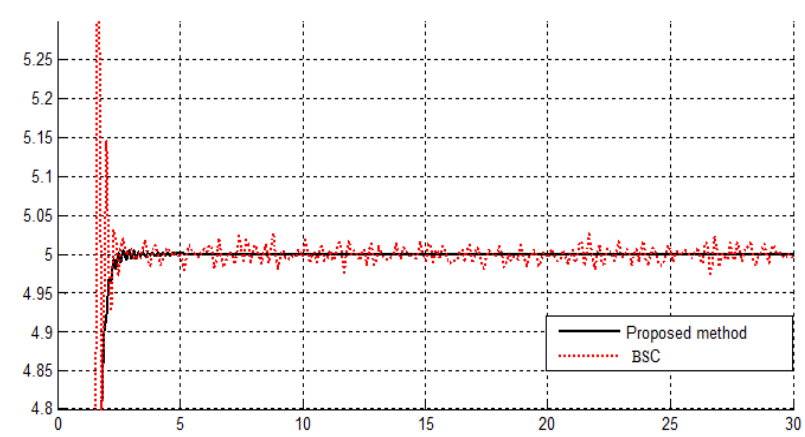

Figure 10: BSC Vs. Proposed method in presence of uncertainty and external disturbance: applied robot manipulator

Based on above graph, pure backstopping controller has many challenges in presence of external disturbance. To eliminate above challenge, proposed methodology is offered.

\section{Conclusion}

On-line tuning modified PI like fuzzy backstopping fuzzy control algorithm for robot manipulators is investigated in this paper. Proposed algorithm utilizes SISO fuzzy system to estimate the cross-coupling effects in robotic manipulator and gets perfect tracking accuracy. Proposed algorithm attenuated the stability and robust problems very well by substituting a nonlinear backstopping methodology and fuzzy logic function for the onlinecontrol term. The number of fuzzy rules in backstopping fuzzy controller is also reduced by the type of inputs in SISO fuzzy systems. Proposed algorithm has predefined adaptation gains in the adaptation laws based on modify PI like fuzzy methodology and increase the stability and robustness in presence of uncertainty.

\section{References}

[1] T. R. Kurfess, Robotics and automation handbook: CRC, 2005.

[2] J. J. E. Slotine and W. Li, Applied nonlinear control vol. 461: Prentice hall Englewood Cliffs, NJ, 1991.

[3] L. Cheng, et al., "Multi-agent based adaptive consensus control for multiple manipulators with kinematic uncertainties," 2008, pp. 189-194. 
[4] J. J. D'Azzo, et al., Linear control system analysis and design with MATLAB: CRC, 2003.

[5] B. Siciliano and O. Khatib, Springer handbook of robotics: Springer-Verlag New York Inc, 2008.

[6] I. Boiko, et al., "Analysis of chattering in systems with second-order sliding modes," IEEE Transactions on Automatic Control, vol. 52, pp. 2085-2102, 2007.

[7] J. Wang, et al., "Indirect adaptive fuzzy sliding mode control: Part I: fuzzy switching," Fuzzy Sets and Systems, vol. 122, pp. 21-30, 2001.

[8] F. Piltan, et al., "Artificial Control of Nonlinear Second Order Systems Based on AFGSMC," Australian Journal of Basic and Applied Sciences, 5(6), pp. 509-522, 2011.

[9] V. Utkin, "Variable structure systems with sliding modes," Automatic Control, IEEE Transactions on, vol. 22, pp. 212-222, 2002.

[10] R. A. DeCarlo, et al., "Variable structure control of nonlinear multivariable systems: a tutorial," Proceedings of the IEEE, vol. 76, pp. 212-232, 2002.

[11] K. D. Young, et al., "A control engineer's guide to sliding mode control," 2002, pp. 1-14.

[12] O. Kaynak, "Guest editorial special section on computationally intelligent methodologies and sliding-mode control," IEEE Transactions on Industrial Electronics, vol. 48, pp. 2-3, 2001.

[13] Samira Soltani \& Farzin Piltan, "Design Artificial Nonlinear Controller Based on Computed Torque like Controller with Tunable Gain", World Applied Science Journal (WASJ), 14 (9): 1306-1312, 2011.

[14] Farzin Piltan, Mohammadali Dialame, Abbas Zare \& Ali Badri,"Design Novel Lookup Table Changed Auto Tuning FSMC:Applied to Robot Manipulator", International Journal of Engineering, 6 (1):25-41, 2012.

[15] Farzin Piltan, Mohammad Keshavarz, Ali Badri \& Arash Zargari,"'Design Novel Nonlinear Controller Applied to RobotManipulator: Design New Feedback Linearization Fuzzy Controller with Minimum Rule Base Tuning Method", International Journal of Robotics and Automation,3 (1):1-12, 2012.

[16] Farzin Piltan, Iman Nazari, Sobhan Siamak, Payman Ferdosali,"Methodology of FPGA-Based Mathematical error-Based Tuning Sliding Mode Controller", International Journal of Control and Automation, 5(1), 89-118, 2012.

[17] Farzin Piltan, Bamdad Boroomand, Arman Jahed \& Hossein Rezaie, "Methodology of Mathematical Error-Based Tuning Sliding Mode Controller", International Journal of Engineering, 6 (2):96-117, 2012.

[18] Farzin Piltan, Sara Emamzadeh, Zahra Hivand, Fatemeh Shahriyari \& Mina Mirazaei, "PUMA560 Robot Manipulator Position Sliding Mode Control Methods Using MATLAB/SIMULINK and Their Integration into
Graduate/Undergraduate Nonlinear Control, Robotics and MATLAB Courses", International Journal of Robotics and Automation, 3(3):106150, 2012.

[19] Farzin Piltan, Ali Hosainpour, Ebrahim Mazlomian, Mohammad Shamsodini, Mohammad H. Yarmahmoudi,'Online Tuning Chattering Free Sliding Mode Fuzzy Control Design: Lyapunov Approach", International Journal of Robotics and Automation, 3(3):77-105, 2012.

[20] Farzin Piltan, Mina Mirzaei, Forouzan Shahriari, Iman Nazari, Sara Emamzadeh, "Design Baseline Computed Torque Controller", International Journal of Engineering, 6(3): 129141, 2012.

[21] Farzin Piltan, Mohammad H. Yarmahmoudi, Mohammad Shamsodini, Ebrahim Mazlomian, Ali Hosainpour, "PUMA-560 Robot Manipulator Position Computed Torque Control Methods Using MATLAB/SIMULINK and Their Integration into Graduate Nonlinear Control and MATLAB Courses", International Journal of Robotics and Automation, 3(3): 167-191, 2012.

[22] Farzin Piltan, Hossein Rezaie, Bamdad Boroomand, Arman Jahed, "Design Robust Backstopping on-line Tuning Feedback Linearization Control Applied to IC Engine", International Journal of Advance Science and Technology, 11:40-22, 2012.

[23] Farzin Piltan, Mohammad R. Rashidian, Mohammad Shamsodini and Sadeq Allahdadi, Effect of Rule Base on the Fuzzy-Based Tuning Fuzzy Sliding Mode Controller: Applied to $2^{\text {nd }}$ Order Nonlinear System", International Journal of Advanced Science and Technology, 46:39-70, 2012.

[24] Farzin Piltan, Arman Jahed, Hossein Rezaie and Bamdad Boroomand, "Methodology of Robust Linear On-line High Speed Tuning for Stable Sliding Mode Controller: Applied to Nonlinear System", International Journal of Control and Automation, 5(3): 217-236, 2012.

[25] Farzin Piltan, Bamdad Boroomand, Arman Jahed and Hossein Rezaie,'Performance-Based Adaptive Gradient Descent Optimal Coefficient Fuzzy Sliding Mode Methodology", International Journal of Intelligent Systems and Applications, vol.4, no.11, pp.40-52, 2012.

[26] M. Bazregar, Farzin Piltan, A. Nabaee and M.M. Ebrahimi, "Parallel Soft Computing Control Optimization Algorithm for Uncertainty Dynamic Systems", International Journal of Advanced Science and Technology, 51, 2013.

[27] Farzin Piltan, M.H. Yarmahmoudi, M. Mirzaei, S. Emamzadeh, Z. Hivand, "Design Novel Fuzzy Robust Feedback Linearization Control with Application to Robot Manipulator", International Journal of Intelligent Systems and Applications, 
vol.5, no.5, pp.1-10, 2013.DOI: 10.5815/ijisa.2013.05.01.

[28] Amin Jalali, Farzin Piltan, M. Keshtgar, M. Jalali, "Colonial Competitive Optimization Sliding Mode Controller with Application to Robot Manipulator", International Journal of Intelligent Systems and Applications, vol.5, no.7, pp.50-56, 2013. DOI: 10.5815/ijisa.2013.07.07.

[29] Afsaneh Salehi, Farzin Piltan, M. Mousavi, A. Khajeh, M. R. Rashidian, "Intelligent Robust Feed-forward Fuzzy Feedback Linearization Estimation of PID Control with Application to Continuum Robot", International Journal of Information Engineering and Electronic Business, vol.5, no.1, pp.1-16, 2013. DOI: 10.5815/ijieeb.2013.01.01.

[30] Amin Jalali, Farzin Piltan, A. Gavahian, M. Jalali, M. Adibi, "Model-Free Adaptive Fuzzy Sliding Mode Controller Optimized by Particle Swarm for Robot manipulator", International Journal of Information Engineering and Electronic Business, vol.5, no.1, pp.68-78, 2013. DOI: 10.5815/ijieeb.2013.01.08.

[31] Farzin Piltan, A. Nabaee, M.M. Ebrahimi, M. Bazregar, "Design Robust Fuzzy Sliding Mode Control Technique for Robot Manipulator Systems with Modeling Uncertainties", International Journal of Information Technology and Computer Science, vol.5, no.8, pp.123-135, 2013. DOI: 10.5815/ijitcs.2013.08.12.

[32] M. M. Ebrahimi, Farzin Piltan, M. Bazregar and A.R. Nabaee, "Intelligent Robust Fuzzy-Parallel Optimization Control of a Continuum Robot Manipulator", International Journal of Control and Automation, 6(3), 2013.

[33] O.R. Sadrnia, Farzin Piltan, M. Jafari, M. Eram and M. Shamsodini, "Design PID Estimator Fuzzy plus Backstopping to Control of Uncertain Continuum Robot", International Journal of Hybrid Information Technology, 6(4), 2013.

[34] Amin Jalali, Farzin Piltan, H. Hashemzadeh, A. Hasiri, M.R Hashemzadeh, "Design Novel Soft Computing Backstopping Controller with Application to Nonlinear Dynamic Uncertain System", International Journal of Intelligent Systems and Applications, vol.5, no.10, pp.93105, 2013. DOI: 10.5815/ijisa.2013.10.12.

[35] M. Moosavi, M. Eram, A. Khajeh, O. Mahmoudi and Farzin Piltan, "Design New Artificial Intelligence Base Modified PID Hybrid Controller for Highly Nonlinear System", International Journal of Advanced Science and Technology, 57, 2013.

[36] S. Zahmatkesh, Farzin Piltan, K. Heidari, M. Shamsodini, S. Heidari, "Artificial Error Tuning Based on Design a Novel SISO Fuzzy Backstopping Adaptive Variable Structure Control" International Journal of Intelligent Systems and Applications, vol.5, no.11, pp.34-46, 2013. DOI: 10.5815/ijisa.2013.11.04.
[37] S. Heidari, Farzin Piltan, M. Shamsodini, K. Heidari and S. Zahmatkesh, "Design New Nonlinear Controller with Parallel Fuzzy Inference System Compensator to Control of Continuum Robot Manipulator",International Journal of Control and Automation, 6(4), 2013.

[38] Farzin Piltan, Mehdi Eram, Mohammad Taghavi, Omid Reza Sadrnia, Mahdi Jafari,"Nonlinear Fuzzy Model-base Technique to Compensate Highly Nonlinear Continuum Robot Manipulator", IJISA, vol.5, no.12, pp.135-148, 2013. DOI: 10.5815/ijisa.2013.12.12.

[39] Amin Jalali, Farzin Piltan, Mohammadreza Hashemzadeh, Fatemeh BibakVaravi, Hossein Hashemzadeh,"Design Parallel Linear PD Compensation by Fuzzy Sliding Compensator for Continuum Robot", IJITCS, vol.5, no.12, pp.97112, 2013. DOI: 10.5815/ijitcs.2013.12.12.

[40] Farzin Piltan, A. Hosainpour, S. Emamzadeh, I. Nazari, M. Mirzaie, "Design Sliding Mode Controller of with Parallel Fuzzy Inference System Compensator to Control of Robot Manipulator", International Journal of Robotics and Automation, Vol. 2, No. 4, December 2013, pp. 149 162.

[41] Farzin Piltan, Mahdi Jafari, Mehdi Eram, Omid Mahmoudi, Omid Reza Sadrnia, "Design Artificial Intelligence-Based Switching PD plus Gravity for Highly Nonlinear Second Order System", International Journal of Engineering and Manufacturing, vol.3, no.1, pp.38-57, 2013.DOI: 10.5815/ijem.2013.01.04.

[42] Farzin Piltan, Sara Emamzadeh, Sara Heidari, Samaneh Zahmatkesh, Kamran Heidari, "Design Artificial Intelligent Parallel Feedback Linearization of PID Control with Application to Continuum Robot", International Journal of Engineering and Manufacturing, vol.3, no.2, pp.51-72, 2013.DOI: 10.5815/ijem.2013.02.04.

[43] Mohammad Mahdi Ebrahimi, Farzin Piltan, Mansour Bazregar, AliReza Nabaee,"Artificial Chattering Free on-line Modified Sliding Mode Algorithm: Applied in Continuum Robot Manipulator", International Journal of Information Engineering and Electronic Business, vol.5, no.5, pp.57-69, 2013. DOI: 10.5815/ijieeb.2013.05.08.

[44] Arman Jahed, Farzin Piltan, Hossein Rezaie, Bamdad Boroomand, "Design Computed Torque Controller with Parallel Fuzzy Inference System Compensator to Control of Robot Manipulator", International Journal of Information Engineering and Electronic Business, vol.5, no.3, pp.66-77, 2013. DOI: 10.5815/ijieeb.2013.03.08.

[45] Mohammad Shamsodini, Farzin Piltan, Mahdi Jafari, Omid reza Sadrnia, Omid Mahmoudi,"Design Modified Fuzzy Hybrid Technique: Tuning by GDO", IJMECS, vol.5, no.8, pp.58-72, 10.5815/ijmecs.2013.08.07. 
[46] Mahdi Mirshekaran, Farzin Piltan,Zahra Esmaeili, Tannaz Khajeaian, Meysam Kazeminasab,"Design Sliding Mode Modified Fuzzy Linear Controller with Application to Flexible Robot Manipulator", IJMECS, vol.5, no.10, pp.53-63, 2013.DOI: 10.5815/ijmecs.2013.10.07.

[47] Meysam Kazeminasab, Farzin Piltan, Zahra Esmaeili, Mahdi Mirshekaran, Alireza Salehi ,"Design Parallel Fuzzy Partly Inverse Dynamic Method plus Gravity Control for Highly Nonlinear Continuum Robot", IJISA, vol.6, no.1, pp.112-123, 2014. DOI: 10.5815/ijisa.2014.01.12.

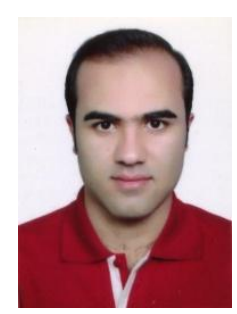

Omid Avatefipour is currently working as a primary researcher in the laboratory of Control and Robotic, Institute of Advance Science and Technology, IRAN SSP research and development Center. His current research interests are in the area of nonlinear control, artificial control system and robotics.

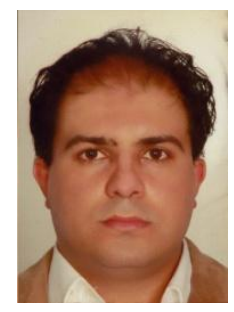

Farzin Piltan was born on 1975, Shiraz, Iran. In 2004 he is jointed Institute of Advance Science and Technology, Research and Development Center, IRAN SSP. Now he is a dean of Intelligent Control and Robotics Lab. In addition to 7 textbooks, Farzin Piltan is the main author of more than 100 scientific papers in refereed journals. He is editorial review board member for 'international journal of control and automation (IJCA), Australia, ISSN: 2005-4297; 'International Journal of Intelligent System and Applications (IJISA)', Hong Kong, ISSN: 2074-9058; 'IAES international journal of robotics and automation, Malaysia, ISSN:2089-4856; 'International Journal of Reconfigurable and Embedded Systems', Malaysia, ISSN:2089-4864. His current research interests are nonlinear control, artificial control system and applied to FPGA, robotics and artificial nonlinear control and IC engine modeling and control.

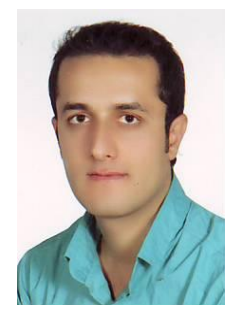

Mahmoud Reza Safaei Nasrabad is currently working as a primary researcher in the laboratory of Control and Robotic, Institute of Advance Science and Technology, IRAN SSP research and development Center. His current research interests are in the area of nonlinear control, artificial control system and robotics.

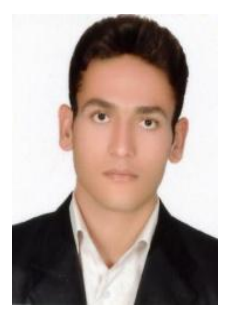

Ghasem Sahamijoo is currently working as a primary researcher in the laboratory of Control and Robotic, Institute of Advance Science and Technology, IRAN SSP research and development Center. His current research interests are in the area of nonlinear control, artificial control system and robotics.

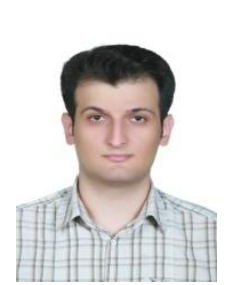

Alireza Khalilian is currently working as a primary researcher in the laboratory of Control and Robotic, Institute of Advance Science and Technology, IRAN SSP research and development Center. His current research interests are in the area of nonlinear control, artificial control system and robotics. 